\title{
The New Two-Dimensional Electron Gas Base HBT (2DEG-HBT): Two-Dimensional Numerical Simulation
}

Patrick D. Rabinzohn, Toshiyuki Usagawa, Hiroshi Mizuta, Member, IEEE, and Ken Yamaguchi, Member, IEEE

\begin{abstract}
The bipolar/FET characteristics of the 2DEG-HBT (TwoDimensional Electron Gas Base p-n-p AIGaAs/GaAs Heterojunction Bipolar Transistor) are analyzed extensively by a two-dimensional numerical simulator based on a drift-diffusion model. For bipolar operations at high collector current densities, it is confirmed that the cutoff frequency $f_{T}$ is determined mainly by the collector transit time of holes and by the charging time of the extrinsic base-collector capacitance $C_{b c}^{\mathrm{EXT}}$. The charging times of the emitter and base regions, and the base transit time are shown to be negligible. A high cutoff frequency $f_{T}(88$ GHz) and current gain $h_{F E}(\mathbf{7 6 0})$ are obtained for an emitter size of 1 $\times 10 \mu \mathrm{m}^{2}$, an undoped collector thickness of $150 \mathrm{~nm}$, and a collector current density $J_{c}$ of $10^{5} \mathrm{~A} / \mathrm{cm}^{2}$.

The FET operation of the same 2DEG-HBT structure shows a threshold voltage $V_{\text {th }}$ of $0.74 \mathrm{~V}$, the transconductance $G_{m}^{\text {max }}$ of 80 $\mathrm{mS} / \mathrm{mm}$, and maximum cutoff frequency $f_{T}^{\max }$ of $15 \mathrm{GHz}$.

Moreover, the buried $\mathrm{SiO}_{2}$ structure for reducing the extrinisic basecollector capacitance $C_{b c}^{\mathrm{EXT}}$ shows an extremely high cutoff frequency $f_{T}$ of $163 \mathrm{GHz}$ and maximum oscillation frequency $f_{\max }$ of $110 \mathrm{GHz}$ for the bipolar mode. The dependence of the device performance on material parameters is analyzed extensively from a device design point of view. Optimization of the epitaxial layer structure enables the design of a higer performance FET (e.g., $G_{m}=235 \mathrm{mS} / \mathrm{mm}$ and $f_{T}=19 \mathrm{GH}$ for $L_{q}=1 \mu \mathrm{m}$ ) without sacrificing the bipolar function.
\end{abstract}

\section{INTRODUCTION}

$\mathrm{T}$ HE 2DEG-HBT (Two-Dimensional Electron Gas Base Heterojunction Bipolar Transistor) [1] is a new functional device, a "bitransistor," operating as both a bipolar transistor and a FET. This bitransistor action is achieved by a new operation principle; the 2DEG at the AlGaAs/GaAs heterointerface acts as either the base of a $p-n-p$ bipolar transistor or the n-channel of a 2DEG-FET [2]

The schematic cross section and the energy-band diagram of the 2DEG-HBT is shown in Fig. 1(a) and (b). The outstanding properties [1] of the proposed 2DEG-HBT are resummarized as follows:

1) Enhanced bipolar performance due to

a) negligible base transit time of holes due to thin $(\sim 10$ $\mathrm{nm}$ ) 2DEG base without neutral region,

b) very low base resistance $r_{b b}^{\prime}$ due to high electron mobility, especially at low temperatures $(77 \mathrm{~K})$.

Manuscript received May 30, 1990; revised August 20, 1990. The review of this paper was arranged by Associate Editor N. Kawamura.

P. D. Rabinzohn was with Philips Research Organization, L. E. P. Limeil-Brevannes, France. He is now with Matra MHS (Wafer Fab.), CP 3008, 44087 Nantes-Cedex 03, France.

$\mathrm{T}$. Usegawa and $\mathrm{K}$. Yamaguchi are with the Central Research Laboratory, Hitachi Ltd., Kokubunji, Tokyo 185, Japan.

H. Mizuta is with Hitachi Cambridge Laboratory, R \& D Centre, Hitachi Europe Ltd., Cavendish Laboratory, Cambridge CB3 OHE, England.

IEEE Log Number 9040646.

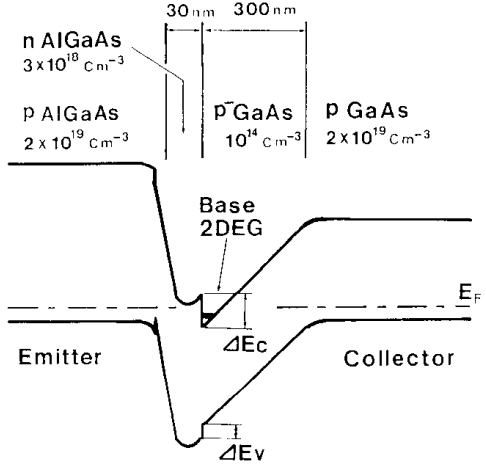

(a)

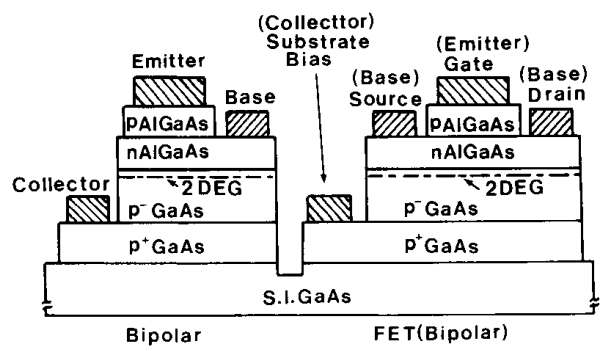

(b)

Fig. 1. The conceptual 2DEG-HBT stucture. (a) Energy-band diagram. (b) Cross-sectional view and monolithic integration of $p-n-p$ HBT and 2DEG-FET by the same epitaxial layer structure.

2) Monolithic integration of 2DEG-FET and p-n-p HBT by the same epitaxial layer structure.

3) Bifunctional operation of bipolar/FET as four-terminal devices.

These new concepts offer significant promise. However, at the early stage of device development [1], some key questions arise: what are the actual operating principles and the electrical characteristics of the 2DEG-HBT, particularly as a bipolar transistor, and can the device operate with high performance as a FET and an HBT? This extensive two-dimensional numerical simulation study addresses these questions, confirming the basic principles of the 2DEG-HBT and demonstrating its effective simultaneous operation as a high-performance p-n-p HBT and junction gate 2DEG-FET by the same structure. Guidelines for 
device design are given by extensive analysis of the dependence of the device performance on material parameters [3].

Finally we comment on the bifunctional operation, i.e., the simultaneous bipolar and FET actions, and the limitation of the present simulator.

\section{Simulated Device Structures}

\section{A. Two-Dimensional Heterostructure Device Simulator}

The present two-dimensional simulator for heterostructure devices was first developed as an engineering tool for laser diodes called HILADIES (HItachi LAser DIodes Engineering Software) [4], and has recently been extended to the design tool for heterostructure electron devices [5]. This simulator, based on the drift-diffusion classical hydrodynamic model [6], has been successfully applied to the analysis of experimental data from the HEMT device. The differences between the present physical modeling and [5] are as follows:

To model the electron velocity overshoot effect, the drift velocity of electrons is described by the camel-like functional form [6]:

$$
V(E)=\frac{\mu_{n 0} E+V_{s}\left(E / E_{c}\right)^{4}}{1+\left(E / E_{c}\right)^{2}}
$$

where $\mu_{n 0}$ is the low-field mobility, $V_{s}$ the saturation velocity, and $E_{c}$ the critical field $\left(4 \times 10^{3} \mathrm{~V} / \mathrm{cm}\right)$. The drift velocity of holes is given by the piecewise linear relation

$$
V(E)= \begin{cases}\mu_{p 0} E, & E \leq V_{s} / \mu_{p 0} \\ V_{s}, & E \geq V_{s} / \mu_{p 0}\end{cases}
$$

where $\mu_{p 0}$ is the low-field mobility of holes, the saturation velocity $V_{s}$ is assumed to be the same for electrons and holes, $10^{7}$ $\mathrm{cm} / \mathrm{s}$. A carrier life time of $1 \mathrm{~ns}$ is assumed for both electrons and holes ( $\left.\tau=\tau_{n}=\tau_{p}=1 \mathrm{~ns}\right)$ unless otherwise specified [4], [5]. We have also introduced a single donor level $D X$ center model as shown in Table I to describe the deep donor density in $\mathrm{n}$-AlGaAs [3], [5].

The material parameters for GaAs, $\mathrm{AlGaAs}$ are listed in Table I. The other mathematical formulas, notations, material parameters, etc., can be found in [4], [5].

\section{B. Simulated "Central" Device Structures}

The 2DEG-HBT structure modeled in this work is shown in Fig. 2. In this work, one of the main concerns is the bipolar/ FET operation by the same structure; the source electrode and the drain electrode also act as the two base electrodes. The simulated device width and the emitter size are fixed at $10 \mu \mathrm{m}$ and $1 \times 10 \mu \mathrm{m}^{2}$, respectively. From an intuitive understanding of the 2DEG-HBT operation, we have defined the "central value" of material parameters for the simulated 2DEG-HBT structure, as specified in Table II.

The simulated device structure (Fig. 2) is a self-aligned structure of the emitter region, the emitter electrode, and the $\mathrm{n}^{+}$ GaAs base contact regions (layers IX), based on a fabricating self-alignment process. The thickness of layers IX is equal to the sum of the thicknesses of layers I to VI. Finally, $\mathrm{SiO}_{2}$ sidewall spacers (layers $X ; 0.15 \mu \mathrm{m}$ thick) provide isolation between the base regions and the emitter and metallugical base; that is, the sidewalls extend to a depth equal to the thickness of

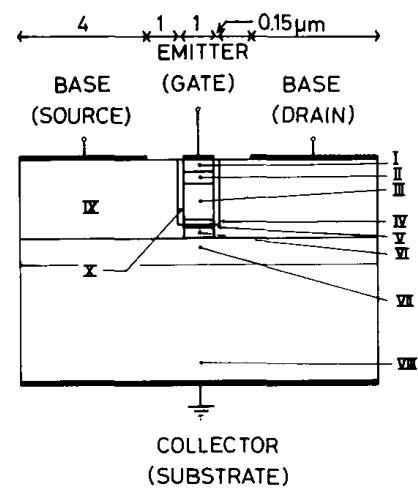

Fig. 2. The simulated 2DEG-HBT structure. The layer structure is described in Table II.

TABLE I

Physical Parameters of $\mathrm{Al}_{x} \mathrm{Ga}_{1}$. As Materials for $x \leqq 0.45$

\begin{tabular}{|c|c|}
\hline Parameter & Formula \\
\hline Dielectric constant & $\epsilon_{r}=13.1-3.0 X$ \\
\hline Electron affinity (eV) & $\chi=4.07-0.6\left(E_{g}^{\Gamma}(x)-\mathrm{E}_{g}^{\Gamma}(0)\right)$ \\
\hline $\begin{array}{l}\text { Bandgaps } \\
\qquad E_{g}^{\Gamma}(\mathrm{eV}) \\
E_{g}^{L}(\mathrm{eV}) \\
E_{g}^{X}(\mathrm{eV})\end{array}$ & $\begin{array}{l}E_{g}^{\Gamma}=1.422+1247 X \\
E_{g}^{L}=1.707+0.642 X \\
E_{g}^{X}=1899+0.125 X+0.143 X\end{array}$ \\
\hline $\begin{array}{l}\text { Electron mass } \\
m_{e}^{\Gamma} / m_{0} \\
m_{e^{L}}^{L} / m \\
m_{e}^{X} / m_{0}\end{array}$ & $\begin{array}{l}m_{e}^{\Gamma} / m_{0}=0.067+0.083 X \\
m_{e}^{L} / m_{0}=0.55+0.12 X \\
m_{e}^{X} / m_{0}=0.85-0.07 X\end{array}$ \\
\hline Hole mass $m_{h} / m_{0}$ & $m_{h} / m_{0}=0.48+0.31 X$ \\
\hline $\begin{array}{l}D X \text { center } \\
\quad \text { level } \\
\Delta E_{D}(\mathrm{meV})\end{array}$ & $\begin{array}{c}\Delta E_{D}=4 \quad 0<X<0.25 \\
=1125 X-277.25 \\
0.25<X<0.37 \\
=-261.54 X+235.77 \\
0.37<X<0.45\end{array}$ \\
\hline
\end{tabular}
AND $T=300 \mathrm{~K}$

TABLE II

Material Parameters for the Simulated "Central" Device STRUCTURES OF THE 2DEG-HBT

\begin{tabular}{rlrrc}
\hline Layer & Material & Al Fraction & $\begin{array}{r}\text { Thickness } \\
(\mathrm{nm})\end{array}$ & $\begin{array}{c}\text { Doping Density } \\
\left(\mathrm{cm}^{-3}\right)\end{array}$ \\
\hline I & p-GaAs & 50 & $10^{20}$ \\
II & p-GaAs & $X_{E}=0.45$ & 150 & $5 \times 10^{19}$ \\
$E$ III & p-AlGaAs & $N_{E}=10^{19}$ \\
$B$ IV & n-AlGaAs & $X_{B}=0.3$ & 25 & $N_{B}=2 \times 10^{18}$ \\
V & p-AlGaAs & $X_{B}=0.3$ & 6 & $10^{14}$ \\
VI & p-GaAs & & $W_{C}=\left\{\begin{array}{r}40 \\
110\end{array}\right.$ \\
$C$ VII & p-GaAs & & 490 & $10^{14}$ \\
VIII & p-GaAs & & & $10^{14}$ \\
IX & $n-G a A s$ & & & $3 \times 10^{18}$ \\
$X$ & $\mathrm{SiO}_{2}$ & &
\end{tabular}

layers I to IV. Thus connection to the base, the 2DEG at the AlGaAs / GaAs interface, is at the contact between $n^{+} \mathrm{GaAs}$ (IX) and layers V and VI. 
For the sake of comparison with typical p-n-p HBT's, the revised p-n-p HBT structure from Sunderland et al. [7] was analyzed as a reference. This $\mathrm{p}-\mathrm{n}-\mathrm{p}$ HBT is described with the help of Fig. 2 and Table II; the metallurgical base (layer IV) and its spacer layer (layer $\mathrm{V}$ ) are replaced by $\mathrm{n}-\mathrm{GaAs}\left(30 \mathrm{~nm}, 3 \times 10^{18}\right.$ $\mathrm{cm}^{-3}$ ) and the lightly doped collector consists of layer VI ( 40 $\left.\mathrm{nm}, 10^{16} \mathrm{~cm}^{-3}\right)$ and layer VII $\left(400 \mathrm{~nm}, 10^{16} \mathrm{~cm}^{-3}\right)$. The other device parameters are the same with the central values as shown in Fig. 2.

The current gain $h_{F E}$ is derived from the variations of the ratio $\Delta I_{C} / \Delta I_{B}$ of collector current $\left(\Delta I_{C}\right)$ to base current $\left(\Delta I_{B}\right)$. The static current gain $\beta=I_{C} / I_{B}$ is used in the final section to discuss the parasitic resistance.

The delay time associated with one region $X$ of the device is given by

$$
\tau_{x}=\left.\frac{\Delta Q_{x}}{\Delta I_{C}}\right|_{V_{C E}}
$$

where $\Delta Q_{x}$ is the incremental excess charge in region $X$ of the simulated device structure, and defined by

$$
\Delta Q_{x}=W \Delta\left(\iint q\left(n-n_{0}\right) d x d y\right)
$$

where $W$ denotes the transistor width of $10 \mu \mathrm{m}$ and $n_{0}$ represents the carrier density in equilibrium. The cutoff frequency is given by

$$
f_{T}=\left.\frac{1}{2 \pi} \frac{\Delta I_{C}}{\Delta Q}\right|_{V_{C E}}
$$

where $\Delta Q$ is the incremental excess charge in the whole simulated device. The device transconductance is calculated by

$$
G_{m}=\left.\frac{\Delta I_{C}}{\Delta V_{B E}}\right|_{V_{C E}}
$$

\section{Operating Principles}

This section concentrates on the device analysis of the "central structure" specified by Table II, to confirm the basic operating principles of the 2DEG-HBT. The specific p-n-p HBT mentioned earlier is also analyzed for comparison.

\section{A. Static Characteristics}

The collector and base currents of the p-n-p HBT and the 2DEG-HBT are plotted in Fig. 3(a) as functions of the emitterbase voltage. The collector current has a similar slope for both devices, with an ideality factor of 1.1. Nevertheless, to obtain the same collector current, the emitter-base junction of the 2DEG-HBT must be forward-biased by $0.2 \mathrm{~V}$ more than the p-n-p HBT, i.e., the collector saturation current of the 2DEG-HBT appears to be smaller ( 500 times). Fig. 3(a) also shows the same ideality factor for the base current of both devices, that is, 1.7 for most of the emitter-base voltage range. Therefore, electron-hole recombination, rather than electron injection into the emitter, dominates the base current of $p-n-p$ HBT's. This is in contrast with the abrupt n-p-n HBT's (see for example [6]).

Thus as shown in Fig. 3(b), the common emitter current gain $h_{F E}$ increases with the collector current density $J_{C}$ until the high injection state is reached. The maximum current gains of 760

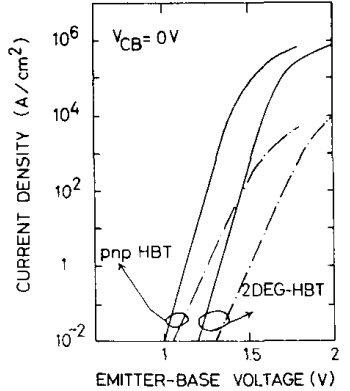

(a)

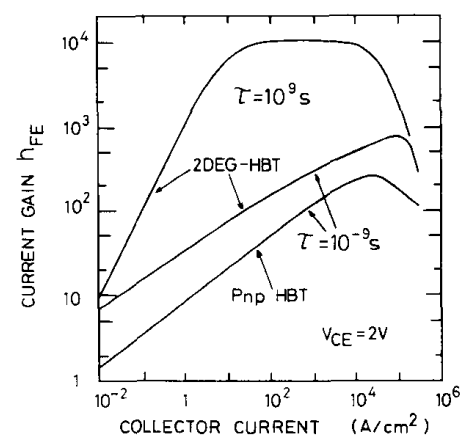

(b)

Fig. 3. Static characteristics of the 2DEG-HBT defined by Fig. 2 and Table II, and the p-n-p HBT specified in the last part of Section II. (a) Collector (solid line) and base (dashed line) currents versus emitter-base voltage. (b) Common emitter current gain $h_{F E}$ versus collector current density, with the carrier lifetime as a parameter.

for the 2DEG-HBT and 280 for the p-n-p HBT are achieved at $10^{5} \mathrm{~A} / \mathrm{cm}^{2}$ and $2 \times 10^{4} \mathrm{~A} / \mathrm{cm}^{2}$, respectively. The higher current gain of the 2DEG-HBT arises from a smaller recombination current.

Since the hole and electron lifetimes were assumed to be equal ( $\tau=1 \mathrm{~ns}$ ) for SRH-type recombination, the wider bandgap metallurgical base of the 2DEG-HBT accounts for the much smaller base current. To confirm the importance of the recombination current, the 2DEG-HBT characteristics shown in Fig. 3(b) were also computed with an artificially long carrier lifetime $\left(\tau=10^{9} \mathrm{~s}\right)$ in all layers. Thus by removing the recombination component, the ideality factor of the base current approaches unity and the $h_{F E}-J_{C}$ curve shows a plateau. The current gain is increased more than tenfold. By setting $\tau=10^{9} \mathrm{~s}$ only in layer IV ( $\mathrm{n}-\mathrm{AlGaAs})$, such a gedanken simulation also establishes that recombination mostly takes place in $\mathrm{n}-\mathrm{AlGaAs}$ because it coincides with the the $h_{F E}-J_{C}$ curve of the above structure with the artificial long carrier life times in all layers.

The carrier concentration profiles are shown in Fig. 4 for the same collector current density. The carrier profile of the $p-n-p$ HBT indicates a neutral base region. On the other hand, the 2DEG-HBT has no neutral base for a forward-biased emitterbase diode. Moreover, the base region is completely depleted of carriers under when not biased. As the n-AlGaAs layer shown in Fig. 4 is not as completely depleted as when biased, the residual electrons in the n-AlGaAs may also act as the base. However, electron injection from $\mathrm{n}$-AlGaAs into the emitter is 


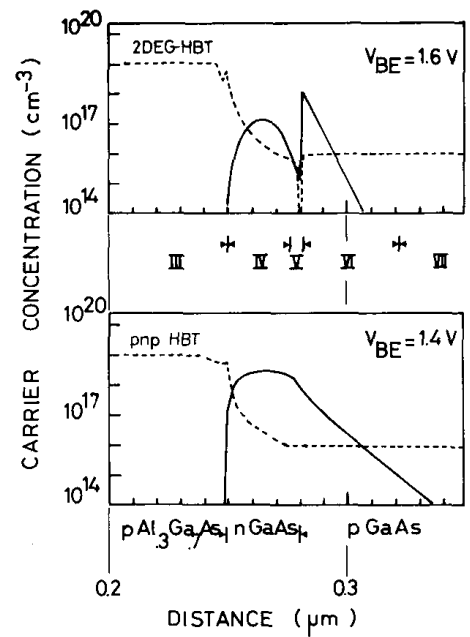

Fig. 4. Electron (solid line) and hole (dashed line) concentration profiles versus distance from the surface in the vicinity of the base, for a collector density of $10^{4} \mathrm{~A} / \mathrm{cm}^{2}$.

suppressed as shown, by designing the emitter layer with a larger Al mole fraction of $\mathrm{p}$-AlGaAs than the metallurgical $\mathrm{n}$-AlGaAs base. Nevertheless, this is not a necessary condition for the 2DEG-HBT to show gain enhancement with respect to the homojunction transistor, as discussed in Section IV. Thus by applying the principle of modulation doping to the base of a bipolar structure, a device without neutral base is truely achieved. The metallurgical base can be considered to be the emitter-base transition layer rather than the actual base (Fig. 4). Therefore, high-frequency and/or high-speed operation is expected as the base transit time is not limited.

\section{B. Frequency Performance Analysis}

Fig. 5 plots the cutoff frequency $f_{T}$ against the collector current density $J_{C}$ for the 2DEG-HBT and the p-n-p HBT. The maximum cutoff frequencies $f_{T}$ are $88 \mathrm{GHz}$ at $J_{C}=4 \times 10^{4}$ $\mathrm{A} / \mathrm{cm}^{2}$ for the 2DEG-HBT, and $19 \mathrm{GHz}$ at $J_{C}=10^{4} \mathrm{~A} / \mathrm{cm}^{2}$ for the p-n-p HBT. The difference in $f_{T}$ results from the different base and collector delay times $\tau_{B}$ and $\tau_{C}$ due to the different structures. This is discussed later. The detailed analysis of the $f_{T}-J_{C}$ curve is performed by subdivision into regional delay times $\tau_{x}$, as defined by (3). The emitter region $E$ consists of p-GaAs (layers I and II) and p-AlGaAs (layer III). The base region $B$ consists of n-AlGaAs metallurgical base (layer IV), $\mathrm{p}$-AlGaAs spacer (layer $\mathrm{V}$ ), and $\mathrm{n}^{+} \mathrm{GaAs}$ base contact regions (layers IX). The collector region $C$ consists of $\mathrm{p}-\mathrm{GaAs}$ (layers VI, VII, and VIII). The analysis of the regional delay times is shown in Fig. 6.

First, it is striking that the delay time associated with the emitter of both devices is always negligible, rising almost linearly with current density to $0.1 \mathrm{ps}$ for the 2DEG-HBT and 0.02 $\mathrm{ps}$ for the $\mathrm{p}-\mathrm{n}-\mathrm{p}$ HBT. In contrast with the conventional design of n-p-n HBT's, the emitter doping level of both devices exceeds the base doping level. Thus the emitter-base transition capacitance charging time would appear in this analysis as a component of the base delay time rather than the emitter delay time for the ongoing analysis. Therefore, the emitter delay time,

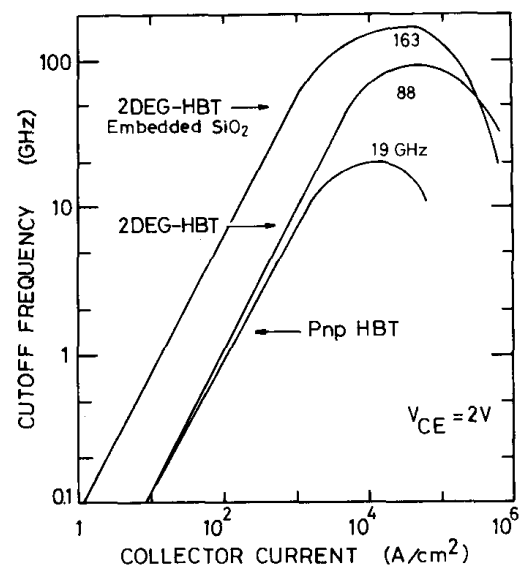

Fig. 5. Cutoff frequency $f_{T}$ versus collector current density $J_{C}$ for the p-n-p HBT, the 2DEG-HBT, and the embedded $\mathrm{SiO}_{2}$ structure of the 2DEGHBT.

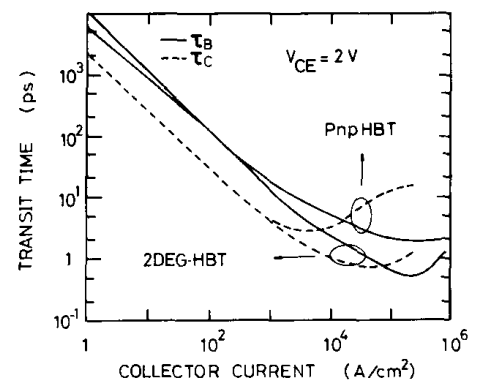

Fig. 6. Base (solid line) and collector (dashed line) delay times versus collector current density for the 2DEG-HBT and p-n-p HBT.

as defined here, is due to the injection of electrons from the base into the emitter, which is so small, that the delay time encountered by minority carriers flowing from emitter to collector is shared by the metallurgical base and collector layers.

The basic principle of the 2DEG-HBT is better understood by comparing the delay times of the two devices at high current densities. The minimum base delay time $\tau_{B}$ of the p-n-p HBT is observed to be $2 \mathrm{ps}$, which can be roughly estimated by the classical formula for the base delay time $\tau_{B}=W_{b}^{2} / 2 D_{p}$ with $W_{b}$ $=30 \mathrm{~nm}$ and $D_{p}=3.1 \mathrm{~cm}^{2} / \mathrm{s}$ for the $3 \times 10^{18} \mathrm{~cm}^{-3} \mathrm{n}-\mathrm{GaAs}$ base. On the other hand, the minimum base delay time $\tau_{B}$ of the 2DEG-HBT is $0.6 \mathrm{ps}$, due to the absence of a neutral base (Fig. 4). The minimum computed collector delay times $\tau_{C}$ for the p-n-p HBT and the 2DEG-HBT are 3.0 and $0.75 \mathrm{ps, \text {respec- }}$ tively, which also can be roughly estinated by a simple formula for the base-collector depletion layer transit time $X_{d} / 2 V_{s}$ where $X_{d}$ denotes the collector depletion layer width, about $440 \mathrm{~nm}$ for the p-n-p HBT and $150 \mathrm{~nm}$ for the 2DEG-HBT.

For a more detailed analysis of the 2DEG-HBT, the regional delay time $\tau_{x}$ is divided into the intrinsic part $\tau^{\text {int }}$ and the extrinsic part $\tau^{\text {ext }}$.

The intrinsic device is the central $1 \mu \mathrm{m} \times 10 \mu \mathrm{m}$ region of Fig. 2. The intrinsic delay time consists of the emitter-base depletion layer (layer IV) delay time $\tau_{b e}^{\text {int }}$, the delay time $\tau_{b}^{\text {int }}$ of layer V, and the base-collector depletion layer (layers VI and 


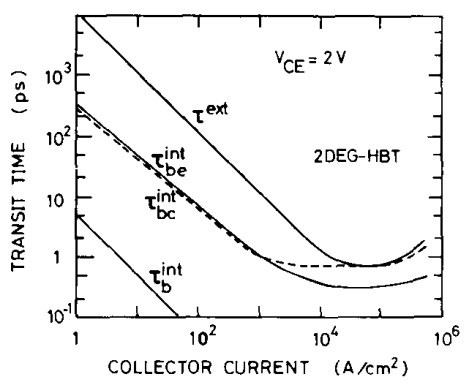

Fig. 7. Extrinsic base-collector charging time $\tau^{\mathrm{ext}}$ (solid line) and intrinsic components of the delay time $\tau_{b e}^{\text {int }}$ (layer IV), $\tau_{b}^{\text {int }}$ (layer V), and $\tau_{b c}^{\text {int }}$ (layers $\mathrm{VI}$ and VII, dashed line) versus collector current density for the structure of Fig. 2 and Table II.

VII) delay time $\tau_{b c}^{\text {int }}$. Such regional analysis of the delay times is shown in Fig. 7.

At low collector current densities, the delay time is dominated by the extrinsic delay time $\tau^{\text {ext }}$, which can be estimated accurately by the ratio of a $110-\mathrm{fF}$ extrinsic base-collector capacitance, in agreement with the geometric capacitance of the extrinsic part of layer VII, to the transconductance $\left(G_{m}=\right.$ $\left.q I_{C} / k T\right)$.

The 2DEG-HBT achieves its minimum delay time at the high current density of $4 \times 10^{4} \mathrm{~A} / \mathrm{cm}^{2}$. In this case, the $1.8-\mathrm{ps} \mathrm{com-}$ puted delay time in Fig. 7 consists of $\tau^{\text {ext }}(=0.75 \mathrm{ps}), \tau_{b e}^{\text {int }}(=$ $0.3 \mathrm{ps})$, and $\tau_{b c}^{\mathrm{int}}(=0.75 \mathrm{ps})$. Even at these large collector current densities, the extrinsic charging time $\tau^{\mathrm{ext}}$ is given accurately by the ratio of capacitance $(110 \mathrm{fF})$ to transconductance $(150 \mathrm{mS})$.

The intrinsic emitter-base transit time $\tau_{b e}^{\text {int }}$ and the base-collector depletion layer transit time $\tau_{b c}^{i n t}$ are well explained by the following simple formulas:

$$
\begin{aligned}
& \tau_{b e}^{\mathrm{int}}=W_{\mathrm{IV}} / V_{s}=0.25 \mathrm{ps} \\
& \tau_{b c}^{\mathrm{int}}=W_{C} / 2 V_{s}=0.75 \mathrm{ps}
\end{aligned}
$$

where $W_{\mathrm{IV}}$ is the thickness of layer IV and $W_{C}$ is the thickness of the lightly doped collector (layers VI and VII). Assuming a triangular concentration profile for the holes in n-AlGaAs (layer IV), as suggested in Fig. 4, and the saturation velocity for the motion of holes through $\mathrm{n}-\mathrm{AlGaAs}, \tau_{b e}^{\text {int }}$ can be represented by (7).

As shown by the regional analysis of the delay times in Fig. 7 , reducing the extrinsic base-collector capacitance $C_{b c}^{\mathrm{EXT}}$ is one of the main issues for realizing the high-performance 2DEGHBT. Therefore, an embedded $\mathrm{SiO}_{2}$ structure was simulated by replacing the extrinsic part of the collector layer VII-VIII (Fig. 2) by $\mathrm{SiO}_{2}$. The computed cutoff frequency $f_{T}$ versus collector current density is shown in Fig. 5 with a maximum cutoff frequency of $163 \mathrm{GHz}$, that is, a minimum delay time of $1 \mathrm{ps,}$ which approaches the intrinsic device performance.

\section{FET Characteristics}

One of the distinct advantages of the 2DEG-HBT is the monolithic integration of high-speed $\mathrm{p}-\mathrm{n}-\mathrm{p}$ transistors and n-channel FET's by the same epitaxial layer structure. From the FET operation point of view, the 2DEG-HBT is basically a p-AlGaAs gate 2DEG-FET with a p-GaAs buffer layer.

To our knowledge, the 2DEG-HBT is the first device with this promising property. The Bipolar Inversion Channel FET

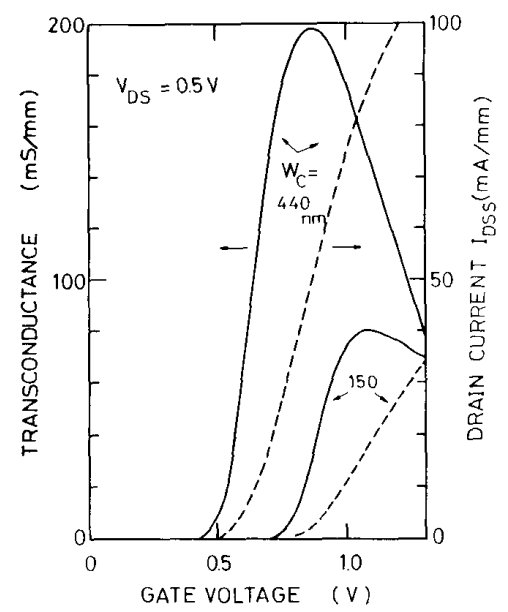

(a)

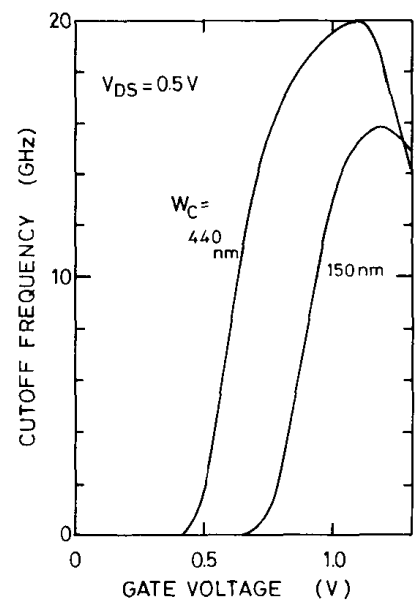

(b)

Fig. 8. FET characteristics of the 2DEG-HBT with $W_{C}$ (Table II) as a parameter. (a) Transconductance (solid line) and drain saturation current (dashed line) versus gate voltage. (b) Cutoff frequency versus gate voltage.

(BICFET) [8] could in principle be considered similarly, but, it has so far been restricted to its bipolar operation, probably to simplify fabrication. Hence the BICFET designed with a planar three terminal (Source, Emitter, Collector) structure has shown serious current crowding problems [9]. On the contrary, the 2DEG-HBT is designed as a four-terminal device, i.e., p-AlGaAs emitter (gate), p-GaAs collector (substrate), and two contacts (source and drain) to the 2DEG base (or n-channel).

The central structure of the 2DEG-HBT, shown in Fig. 2 and Table II, is simulated as a junction-gate FET with a p-GaAs buffer layer. The FET characteristics (transconductance $G_{m}$, gate capacitance $C_{G}$, and cutoff frequency $f_{T}$ ) are simulated by the same definitions as in [5].

The transconductance $G_{m}$, the source-drain saturation current $I_{D S S}$, and the cutoff frequency $f_{T}$ are shown in Fig. 8(a) and (b) for the central structure $\left(W_{C}=150 \mathrm{~nm}\right)$ and the wider collector structure ( $W_{C}=440 \mathrm{~nm}$ ). The difference between the FET performances comes from the fact that the built-in potential from the collector p-GaAs (layer VIII) reduces the 2DEG sheet density. 


\section{Influence of the Device Parameters}

To investigate the dependence of the device performance on the material parameters, each device parameter was individually varied from the "central value" in Fig. 2 and Table II. Simulations were performed changing the Al mole fraction, the doping level, and the thicknesses of the emitter (layer III), metallurgical base (layer IV), and collector (layer V-VI).

\section{A. Emitter and Base Al Mole Fraction}

As stated before, the primary effect of designing the p-AlGaAs emitter layer (III) with a higher $\mathrm{Al}$ mole fraction than the $\mathrm{n}$-AlGaAs base layer (IV) is to prevent electron injection from the $\mathrm{n}-\mathrm{AlGaAs}$ into the emitter. Hence the base current is reduced to its recombination component. This is shown by the current gain-collector current density $\left(h_{F E}-J_{C}\right)$ curve in Fig. 9. In contrast with the device reported so far (emitter $\mathrm{Al}$ content $X_{E}=0.45$ ), the 2DEG-HBT with $X_{E}=X_{B}=0.3$ shows a flat current gain, that is, a base current ideality factor close to unity. Therefore, electron injection into the emitter reduces the maximum current gain to 115 . However, this $h_{F E}$ is still twice that of the homojunction $\mathrm{Al}_{0.3} \mathrm{Ga}_{0.7}$. As p-n-p bipolar transistor and ten times larger than that of the GaAs $p-n-p$ bipolar transistor. This result is in qualitative agreement with the basic principle of the 2DEG-HBT.

The cutoff frequency-collector current density characteristics of the 2DEG-HBT with $X_{E}=0.3$ is similar to that of Fig. 5 ( $X_{E}=0.45$ ) but saturates at $54 \mathrm{GHz}$, that is, $\tau_{b e}^{\text {int }}=0.55 \mathrm{ps}$ and $\tau_{b c}^{\text {int }}=1.35 \mathrm{ps}$. This can be explained by a hole velocity smaller than the saturation velocity obtained for $X_{E}=0.3$. Hence the emitter-base heterojunction may also act as a hot hole launcher.

The maximum gain $h_{F E}^{\max }$ of the 2DEG-HBT and the current density associated with this $h_{F E}^{\max }$ are plotted in Fig. 10 as functions of the n-AlGaAs Al mole fraction $X_{B}$. The $h_{F E}^{\max }-X_{B}$ curve is strongly correlated with the dependence of $X_{B}$ on the deep donor (i.e., $D X$ center) energy $\Delta E_{D}$ (Table I). As $\Delta E_{D}$ increases, the ionized donor density as well as the free electron concentration in $\mathrm{n}$-AlGaAs decrease. This reduces the recombination current. The importance of the $D X$ centers to the base current was also checked by simulating the 2DEG-HBT $\left(X_{B}=\right.$ 0.3 ) with a fully ionized impurity model. As shown in Fig. 10, the computed value of $h_{F E}^{\max }$ is 215 instead of 760 for the deep donor model. The reported correlation mentioned above and the band diagram suggests that the electron quasi-Fermi level is pinned at the deep donor energy.

In contrast with the dc characteristics, the cutoff frequency of the 2DEG-HBT is constant for $X_{B}$ between 0.2 and 0.4 .

\section{B. Emitter and Base Doping Level}

The $h_{F E}-J_{C}$ curve is shown in Fig. 11 with the emitter doping level $N_{E}$ as a parameter. For emitter doping less than $10^{18} \mathrm{~cm}^{-3}$, electron injection into the emitter dominates. Moreover, the emitter series resistance limits the current-handling capability. The corresponding low value of the device transconductance, $40 \mathrm{mS}$, also reduces the maximum cutoff frequency to $39 \mathrm{GHz}$. It is therefore highly desirable to design the emitter with a higher doping level than the base, as for any bipolar transistors. However, this design principle is usually modified for n-p-n HBT's [10], mostly because of the limited doping level achievable for $n$-AlGaAs. One of the advantages of p-n-p GaAs HBT's over $n-p-n$ GaAs HBT's is the high doping capability of the emitter region.

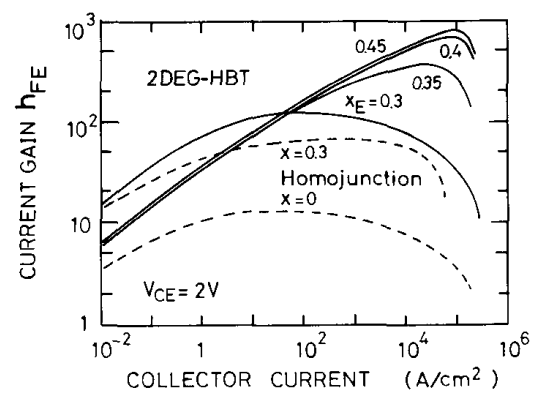

Fig 9. Common emitter current gain versus collector current density for the 2DEG-HBT (solid line) with the emitter Al content $X_{E}$ as a parameter. The dashed lines are for p-n-p homojunction transistors with Al contents $X$ $=0.3$ and 0.0 . Other parameters are as in Table II.

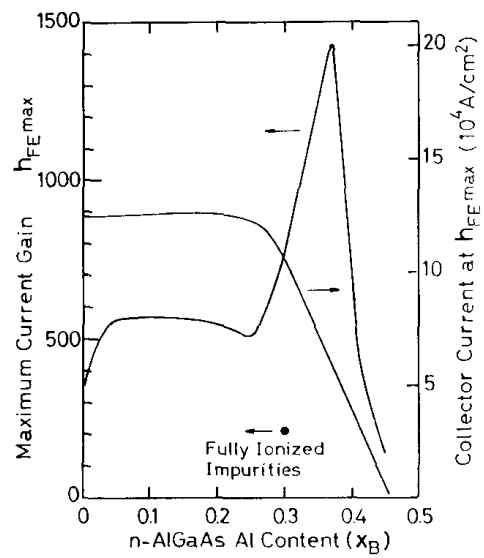

Fig. 10. Maximum current gain $h_{F E}^{\max }$ and corresponding collector current density versus $n$-AlGaAs Al content $X_{B}$. Other parameters are as in Table II. The dark point $\bullet$ was calculated with a fully ionized impurity model for $X_{B}=0.3$

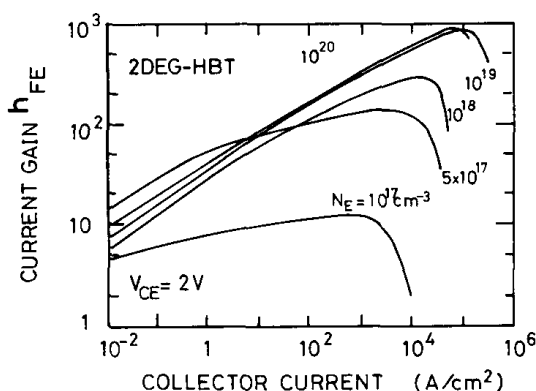

Fig. 11. $h_{F E}-J_{C}$ characteristics of the 2DEG-HBT with the emitter $N_{E}$ (layer III) doping level as a parameter. Other parameters are as in Table II.

Fig. 12 plots the $h_{F E}-J_{C}$ characteristic with the n-AlGaAs doping level $N_{B}$ of the metallurgical base as a parameter. At low doping levels, the computed current gain appears to be as high as $10^{5}$ for a small emitter-base forward bias. This phenomenon is accompanied by a large collector current showing an ideality factor of around 15 . In this condition the device could be described as a punchthrough diode. For the enhancement- 


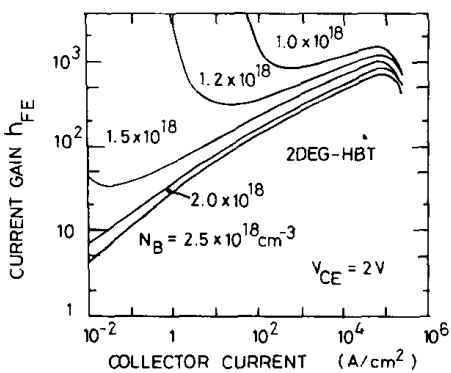

Fig. 12. $h_{F E}-J_{C}$ characteristics of the 2DEG-HBT with the metallurgical base (layer IV) doping level $N_{B}$ as a parameter.

type 2DEG-HBT reported here, this phenomenon always exists, e.g., only at very small current densities for the structure of Table II. The low doping limit of the metallurgical base, $\mathrm{n}$-AlGaAs, is similar to that of devices such as the Bipolar Inversion Channel FET (BICFET) [8] or the Inversion Base Transistor (IBT) [11]. In this case, even though an extremely large current gain $h_{F E}$ is expected at low collector current densities, this simulation results show that the emitter and collector will be in the punchthrough state.

In the investigated range of $N_{B}\left(1-3 \times 10^{18} / \mathrm{cm}^{3}\right)$, the cutoff frequency is not affected at high current densities, above $10^{3}$ $\mathrm{A} / \mathrm{cm}^{2}$. However, the rise of $f_{T}$ with $J_{C}$ is steeper with the punchthrough effect.

\section{Collector Thickness}

The $h_{F E}-J_{C}$ curve of the 2DEG-HBT is unchanged at low current by variations in the lightly-doped collector (layers VI and VII) width $W_{C}$. The only effect of increasing $W_{C}$ is a reduction in the current density at which $h_{F E}$ starts to fall; this is attributed to the Kirk effect. Thus a smaller $h_{F E}^{\max }$ is observed, e.g., 380 for $W_{C}=440 \mathrm{~nm}$.

The cutoff frequency characteristics of the 2DEG-HBT are given in Fig. 13 for $W_{C}=100,150$, and $440 \mathrm{~nm}$. The shift of the curve toward lower current densities as $W_{C}$ increases indicates that the extrinsic base-collector capacitance is reduced For $W_{C}=440 \mathrm{~nm}, f_{T}$ saturates at $23 \mathrm{GHz}$ as the intrinsic basecollector transit time $\tau_{b c}^{\text {int }}$ increases to 5 ps. From the device design point of view, this cutoff frequency of the 2DEG-HBT with the $W_{C}=440 \mathrm{~nm}$ structure should be compared with the reference p-n-p HBT which has a maximum $f_{T}$ of $19 \mathrm{GHz}$ defined by the last part of Section II. For $W_{C}=100 \mathrm{~nm}$, the improvement in $\tau_{b c}^{\text {int }}$ is balanced by an increase in $\tau_{b e}^{\text {int }}(1 \mathrm{ps})$. Thus $f_{T}$ shows a small improvement at most: $92 \mathrm{GHz}$ compared to 88 $\mathrm{GHz}$ for $W_{C}=150 \mathrm{~nm}$.

\section{FET Characteristics and Bifunctional Operations}

For the FET operation of the 2DEG-HBT, the main points of interest are the effects of the $p$-buffer layer and $p-n$ junction gate on the FET performance.

In the normal design of 2DEG-HBT, the p-AlGaAs gate doping level $N_{E}$ is much larger than the n-AlGaAs doping level $N_{B}$. However, a decrease in the gate doping level to $N_{E}=1 \times$ $10^{18} / \mathrm{cm}^{3}$ strongly affects the device performance; the threshold voltage $V_{T}$ becomes $-0.55 \mathrm{~V}$ (depletion-type FET) and the maximum transconductance $G_{m}^{\max }$ falls to $55 \mathrm{mS} / \mathrm{mm}$. When the p-AlGaAs gate doping level $N_{E}$ is smaller than the n-AlGaAs doping level $N_{B}$, the depletion layer extends more deeply into

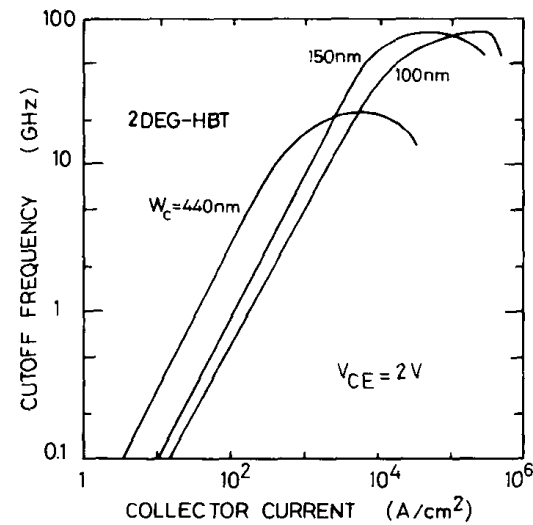

Fig. 13. $f_{T}-J_{C}$ characteristics of the 2DEG-HBT with the lightly doped collector thickness $W_{C}$ as a parameter.

the p-AIGaAs layer so that the FET performance degrades further.

The characteristics of the FET operation of the 2DEG-HBT are given in Fig. 14(a) and (b) with the $\mathrm{n}-\mathrm{AlGaAs}$ doping level $N_{B}$ as a parameter. The threshold voltages $V_{T}$ are $0.04,0.74$, and $0.89 \mathrm{~V}$ for $N_{B}=3,2$, and $1.2 \times 10^{18} / \mathrm{cm}^{3}$, respectively. $G_{m}$ and $f_{T}$ are unchanged by increasing $N_{B}$ from 2 to $3 \times$ $10^{18} / \mathrm{cm}^{3}$. Most importantly, the donor neutralization process has a decreasing influence on the formation of $2 D E G$, as the doping level is lowered below $2 \times 10^{18} / \mathrm{cm}^{3}$. Thus a higher maximum transconductance $(235 \mathrm{mS} / \mathrm{mm})$ and cutoff frequency $(19 \mathrm{GHz})$ are obtained for $1.2 \times 10^{18} / \mathrm{cm}^{3}$.

The 2DEG-HBT is the first device to operate simultaneously as a FET and a bipolar. The bifunctional mode is obtained by biasing both the gate (or emitter) and the drain (or right-hand side base). We first consider the effect of a large applied gate voltage on the FET characteristics for the embedded $\mathrm{SiO}_{2}$ structure. The source-drain saturation current $I_{d s s}$ and the transconductance $G_{m}$ are shown in Fig. 15(a) as functions of the gate voltage. For $V_{G} \geqq 1.9 \mathrm{~V}$, the transconductance $G_{m}$ reaches a plateau of $2.4 \mathrm{~S} / \mathrm{mm}$. In this region, the source supplies additional electrons to electrically neutralize the large number of holes injected into the heterointerface. The carrier concentration profiles computed for $V_{G}=2 \mathrm{~V}$ explain this situation in Fig. 15(b). The gate capacitance also steadily increases; the corresponding cutoff frequency $f_{T}$ has a peak value of $7.5 \mathrm{GHz}$ for $V_{G}=1.9 \mathrm{~V}$.

Regarding the bifunctional operation, biasing the drain with respect to the source enables injection of electrons into the channel in excess of the equilibrium 2DEG density. Charge neutrality is maintained because of the large hole flow perpendicular to the electron flow. Consequently, a neutral charge region with negligible electric field is formed in the lightly doped p-GaAs layer.

The current characteristics are very similar to the bipolar $I-V$ curves of Fig. 3(a), with ideality coefficients of 1.15 and 1.50 for the collector and base currents, respectively. Indeed, with the preceding definition of the base current, the collector and base currents obtained for $V_{D S}=0.5 \mathrm{~V}$ and $V_{B E}=X \mathrm{~V}$ are exactly equal to the collector and base currents computed for $V_{D S}=0 \mathrm{~V}, V_{B E}=X-0.12 \mathrm{~V}$. Hence, the $h_{F E}-J_{C}$ characteristics remain unchanged by applying a drain bias. The $f_{T}-J_{C}$ curve is also similar to that of the bipolar mode (no drain bias) 


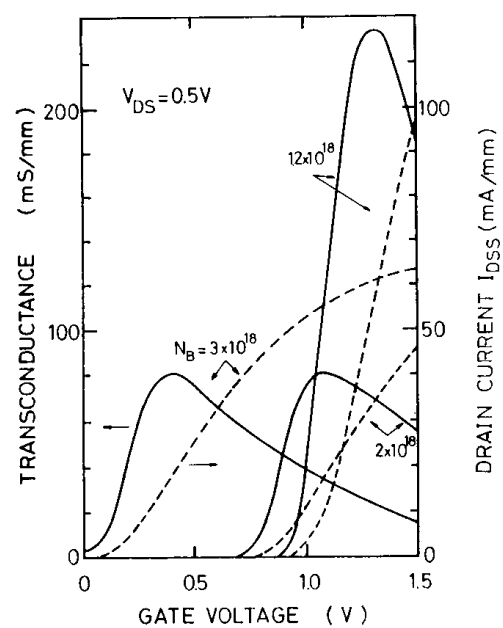

(a)

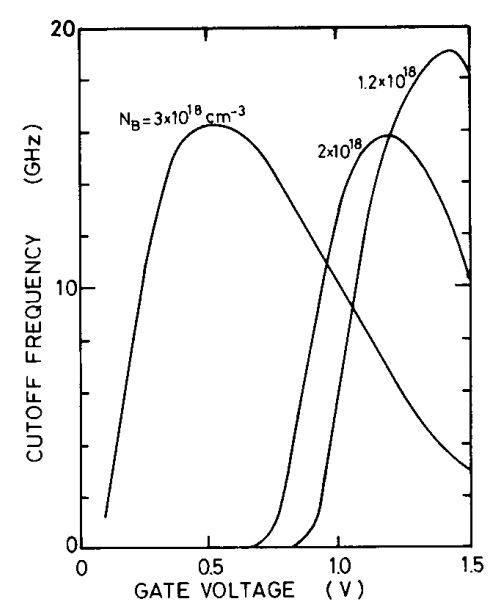

(b)

Fig. 14. FET characteristics of the 2DEG-HBT with the $n$-AIGaAs doping level $N_{B}$ as a parameter. (a) Transconductance (solid line) and drain saturation current (dashed line) versus gate voltage. (b) Cutoff frequency versus gate voltage.

shown in Fig. 5. However, the maximum cutoff frequency is $225 \mathrm{GHz}$ for collector current densities of $2 \times 10^{4}$ to $10^{5}$ $\mathrm{A} / \mathrm{cm}^{2}$, instead of $163 \mathrm{GHz}$ for the bipolar mode. The emitterto-collector delay time is less than that of the standard bipolar operation, i.e., 0.75 ps versus 1 ps.

This bifunctional operation could in principle be applied to any bipolar transistors. However, effective modulation of the channel (base) in the FET mode would prevents protection against punchthrough of the neutral base (channel) in the bipolar mode. This limitation does not apply to the 2DEG-HBT as it has no neutral base.

\section{Discussions}

This section discusses the base resistance $r_{b b}^{\prime}$, the maximum oscillation frequency $f_{\max }$, the limitation of the classical simulator.

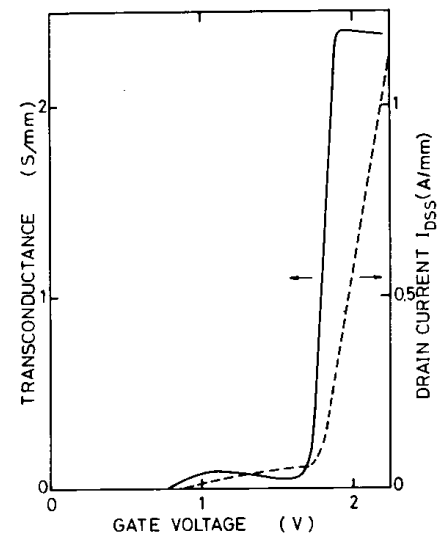

(a)

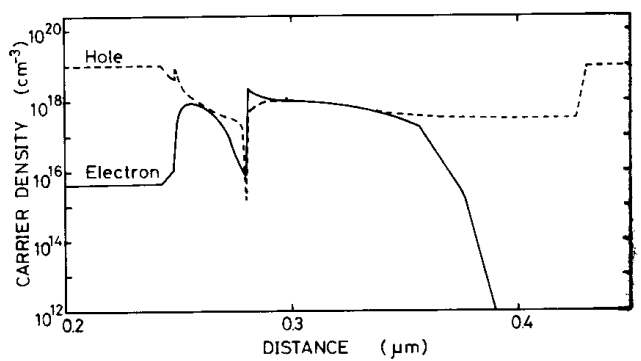

(b)

Fig. 15. FET interpretation of the bifunctional mode: extension of the FET characteristics (Fig. 8) towards larger gate voltages. (a) Transconductance (solid line) and drain saturation current (dashed line) versus gate voltage. (b) Electron (solid line) and hole (dashed line) concentration profiles versus distance from the mid-gate area near the base, for $V_{G}=2 \mathrm{~V}$ and a collector current density of $5 \times 10^{5} \mathrm{~A} / \mathrm{cm}^{2}$.

\section{A. Base Resistance $r_{b b}^{\prime}$ and Maximum Oscillation Frequency $f_{\max }$}

One of the advantages of the bipolar transistor is the base resistance $r_{b b}^{\prime}$, which depends on the extrinsic shape of the transistor.

The main task of the device design is to achieve a device structure with a low base resistance $r_{b b}^{\prime}$, keeping the current gain $h_{F E}$ and $f_{T}$ as high as possible.

In this section we comment on a simple estimation of the base resistance $r_{b b}^{\prime}$ with an assumption that the intrinsic base resistance at high collector currents can be estimated to be $10 \Omega$ from the $1-\mathrm{k} \Omega / \square$ 2DEG sheet density. This assumption gives an estimated base resistance $r_{b b}^{\prime}$ of $20 \Omega$, which value is one order of magnitude smaller than that of the advanced Si bipolar transistors [12].

Assuming $20 \Omega$ for the base transistance $r_{b b}^{\prime}$, the maximum oscillation frequencies $f_{\max }$ can be estimated to be $40 \mathrm{GHz}$ for the "central structure" of Fig. 2 and Table II, and $103 \mathrm{GHz}$ for the $\mathrm{SiO}_{2}$-embedded structure, in which the maximum oscillation frequency $f_{\max }$ can be estimated by the conventional formula

$$
f_{\max }=\sqrt{f_{T} / 8 \pi r_{b b}^{\prime} C_{B C}} .
$$

The base-collector capacitances $C_{B C}$ are 110 and $30 \mathrm{fF}$ for both structures, respectively. As the intrinsic base-collector capac- 
itance $C_{B C}^{i}$ for the "central structure" is $7.7 \mathrm{fF}$, the real basecollector capacitance $C_{B C}$ can be reduced by shrinking the extrinsic base-collector region. In this case, the intrinsic maxi$\operatorname{mum} f_{\max }$ is almost $200 \mathrm{GHz}$.

\section{B. Limitations of the Present Simulator}

1) Quantum Effects: One of the main questions regarding the applicability of the classical simulator to the 2DEG-HBT is to what extent can the simulator quantitatively describe the vertical and horizontal motion of the 2DEG.

As was previously noted in Section II, the present simulator has successfully demonstrated [5] that the sheet number of the 2DEG at the n-AlGaAs/undoped GaAs heterointerface can be quantitatively described by introducing the $D X$ center model in $\mathrm{n}$-AlGaAs. It also shows that the horizontal motion of the 2DEG along the heterointerface is well described to explain the experimental data of the HEMT characteristics. However, the classical simulator cannot describe the real shape of the vertical distribution of electrons at the $\mathrm{AlGaAs} / \mathrm{GaAs}$ heterointerface due to the quantum effects [13]. One of the distinctive features of the 2DEG-HBT is that the base is formed in the undoped semiconductor layer (VI) of Fig. 2 and Table II, which is separate from the original doped layer, n-AlGaAs, (IV). The 2DEG-HBT could control the vertical distribution of the 2DEG by the electric field through the base-collector voltage $V_{B C}$. However, the quantum-mechanical description of the vertical distribution of the 2DEG is inevitably beyond the classical description.

The vertical motion of the 2DEG through the AlGaAs/GaAs heterointerface cannot be quantitatively described by the classical simulator because the transition probabilities of electrons and holes through the heterointerface should be determined by quantum mechanics. Generally speaking, the real base current $I_{B}$ and the current gain $h_{F E}$ are thought to be affected by the recombination rate of the minority carriers at the surface or junction, and the transition rate through the heterointerface, so that it is not an easy task to simulate quantitatively the real base current or the current gain.

2) Overshoot Effect of Holes: The present classical simulator models the behavior of light holes and heavy holes under the high electric field by introducing one saturation velocity $V_{s}$ for both holes. The motion of light and heavy holes under the high electric field is a key factor in the bipolar operation of the 2DEG-HBT's. In the case of n-p-n HBT's, the specific collector structure [14] has demonstrated the overshoot effect of electrons. The overshoot effect of the light hole was recently reported in a low-temperature hot hole transistor structure [15]. If the overshoot effect of holes takes place at room temperature, this simulator could incorporate phenomenologically the overshoot effect by assigning a larger value to the saturation velocity $V_{s}$.

Regarding the surface recombination effects on the base current, the experiment [16] and the simulation [17] showed that the surface recombination effect is the limiting factor of current gain $h_{F E}$. Even though full understanding of the surface recombination is incomplete, this simulator can incorporate phenomenologically the recombination term in the surface or junction area.

\section{Conclusions}

This study has demonstrated the device concept and the bipolar operation of the two-dimensional electron gas base HBT (2DEG-HBT). Simulation shows the base transit time to be negligible and the emitter-to-collector delay time is limited mostly by the base-collector depletion layer transit time. For the bipolar operation, a cutoff frequency $f_{T}$ of $163 \mathrm{GHz}$ and maximum oscillation frequency $f_{\max }$ of $100 \mathrm{GHz}$ are predicted for an ideal device structure with an $\mathrm{SiO}_{2}$-embedded structure with an emitter size of $1 \mu \mathrm{m} \times 10 \mu \mathrm{m}$. Optimization of the epitaxial layer structure enables the tradeoffs for the design of a higher performance FET (e.g., $G_{m}=235 \mathrm{mS} / \mathrm{mm}$ and $f_{T}=$ $19 \mathrm{GHz}$ for $L_{g}=1 \mu \mathrm{m}$ ) without sacrificing of the bipolar function.

As for the bifunction operation, biasing the drain with respect to the source, the bipolar mode shows an extremely high cutoff frequency $f_{T}$ of 225 for the embedded $\mathrm{SiO}_{2}$ structure.

The 2DEG-HBT can easily achieve monolithic integration on p-n-p HBT and 2DEG-FET (or HEMT: Schottky-gate 2DEG-FET) by the same epitaxial multi-layer structures. This merged integrated structures of 2DEG-HBT are promising for future high-speed and low-power BiCMOS-like GaAs LSI's.

\section{ACKNOWLEDGMENT}

The authors wish to thank Dr. H. Matsumura for his continuing encouragement.

\section{REFERENCES}

[1] T. Usagawa, S. Goto, T. Mishima, M. Yamane, M. Kobayashi, M. Kawata, and S. Takahashi, "A new two-dimensional electron gas base transistor (2DEG-HBT)," in IEEE IEDM Tech. Dig., pp. $78-81$, Dec. 1987

[2] T. Mimura, S. Hiyamizu, T. Fujii, and K. Nanbu, "A new field effect transistor with selectivity doped GaAs $/ \mathrm{Al}_{x} \mathrm{Ga}_{1-x}$ As heterojunctions," Japan J. Appl. Phys., vol. 19, pp. L225-L227, 1980.

[3] T. Ishikawa, J. Saito, S. Sasa, and S. Hiyamizu, "Electrical properties of $\mathrm{Si}$-doped $\mathrm{Al}_{x} \mathrm{Ga}_{1-x}$ As layers grown by MBE, ' $J a$ pan J. Appl. Phys., vol. 21, pp. L675-L676, 1082.

[4] T. Ohtoshi, K. Yamaguchi, C. Nagaoka, T. Uda, Y. Murayama, and N. Chinone, "A two-dimensional device simulator of semiconductor lasers," Solid-State Electron., vol. 21, pp. 627-638, 1987.

[5] H. Mizuta, K. Yamaguchi, M. Yamane, T. Tanoue, and S. Takahashi, "Two-dimensional numerical simulation of Fermi-level pinning phenomena due to $D X$ centers in $\mathrm{AlGaAs} / \mathrm{GaAs}$ HEMT's," IEEE Trans. Electron Devices, vol. 36, pp. 2307$2314,1989$.

[6] J. Yoshida, M. Kurata, K. Morizuka, and A. Hojo, "Emitterbase bandgap grading effects on AlGaAs/GaAs heterojunction bipolar transistor characteristics," IEEE Trans. Electron Devices, vol. ED-32, pp. 1714-1721, 1985.

[7] D. A. Sunderland and P. D. Dapkus, "Optimizing n-p-n and p-n-p heterojuction bipolar transistors for speed,' IEEE Trans. Electron Devices, vol. ED-34, pp. 367-377, 1987.

[8] G. W. Taylor and J. G. Simmons, "Bipolar inversion channel field effect transistor," IEEE Trans. Electron Devices, vol. ED-32, pp. 2345-2367, 1985

[9] M. Meyyapan, J. P. Kreskovsky, and H. L. Grubin, "Numerical Simulation of an AlGaAs / GaAs bipolar inversion channel field effect transistor," Solid-State Electron., vol. 26, pp. 1023-1030, 1988.

[10] H. Kroemer, "Heterojunction bipolar transistors and integrated circuits," Proc. IEEE, vol. 70, pp. 64-76, 1982.

[11] K. Matsumoto, Y. Hayashi, T. Kojima, T. Nagata, and T. Yoshimoto, "Integration of GaAs SISFET and GaAs inversion-base bipolar transistor," in Extended Abstracts 20th Conf. on Solid State Devices and Materials, pp. 531-534, 1988.

[12] T. Nakamura, K. Ikeda, N. Kakazato, K. Washio, M. Namba, and T. Hayashida, "63 ps ECL circuits using advanced SICOS technology," in IEEE IEDM Tech. Dig., pp. 472-475, Dec. 1986.

[13] T. Ando, "Self-consistent results for a GaAs/AlGaAs heterojunction. I. Subband structure and light-scattering spectra," $J$. Phys. Soc. Japan, vol. 51, pp. 3893-3899, 1982. 
[14] T. Ishibashi and Y. Yamauchi, "A possible near-ballistic collection in an AIGaAs/GaAs HBT with a modified collector structure," IEEE Trans. Electron Devices, vol. 35, pp. 401-404, 1988.

[15] M. Heiblum, "Ballistic transport of electrons and holes," in IEEE IEDM Tech. Dig., pp. 822-825, Dec. 1988

[16] O. Nakajima, K. Nagata, H. Ito, T. Ishibashi, and T. Sugeta, "Emitter-base junction size effect on current gain $\mathrm{Hf}_{e}$ of AlGaAs/GaAs heterojunction bipolar transistors," Japan J. Appl. Phys., vol. 24, pp. L596-598, 1985.

[17] Y. Someya Hiraoka, J. Yoshida, and M. Azuma, "Two-dimensional analysis of emitter-size effect on current gain for $\mathrm{Ga}$ AlAs/GaAs HBT's," IEEE Trans. Electron Devices, vol. ED-34, pp. 721-725, 1987.

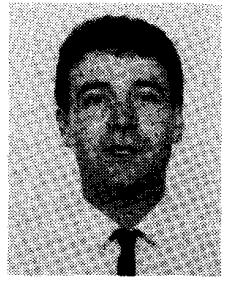

Patrick D. Rabinzohn was born in Belley, France, on February 19, 1958. He graduated as an Engineer from the "Ecole Supérieure d'Electricité," Gif-sur-Yvette, France, in 1981 , and received the $\mathrm{Ph} . \mathrm{D}$. degree in material science from "Pierre et Marie Curie"' University, Paris, France, in 1983

In 1984, he joined the "Laboratorie d'Electronique Philips," Limeil-Brévannes, France. From 1984 to 1987 he worked in the research and development of GaAs MMIC's and dry etching processing for GaAs IC's technologies. As an Exchange Researcher from the Philips Research Organization, he was in 1988 a Visiting Researcher at the Central Research Laboratory, Hitachi Ltd., Tokyo, Japan, where he worked on research and development of new compound semiconductor electron devices. In 1989, at L.E.P., he was supervising research and development on III-V devices and IC's processing. In November 1989, he joined Matra MHS, Nantes, France, as a member of the engineering department.

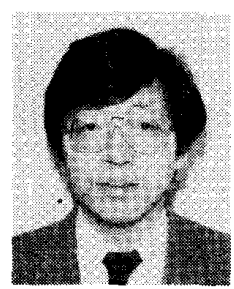

Toshiyuki Usagawa was born in Yamaguchi, Japan, in 1953. He received the B.S., M.S., and Ph.D. degrees, all in physics, from the University of Tokyo, Tokyo, Japan, in 1976, 1978 , and 1981 , respectively. His thesis subject was the theoretical work on Spin Dynamics with Relaxation of Superfluid ${ }^{3} \mathrm{He}$.

In 1981, as a Research Fellow of Japan Society for the Promotion of Science, he was involved in the group-theoretical analysis of the antiferromagnetic ordered nuclear spins of solid
${ }^{3} \mathrm{He}$. He joined the Central Research Laboratory, Hitachi, Ltd., in 1982 , and has been working in the field of GaAs / AlGaAs heterojunction high-speed devices. He has been involved in the deep-recessed gate structure of 2DEG-FET and 2DEG-HBT. His current interests are in mesoscopic electronics and the electrooptic merged devices of the III-V materials.

Dr. Usagawa is a member of the Physical Society of Japan and the Japan Society of Applied Physics.

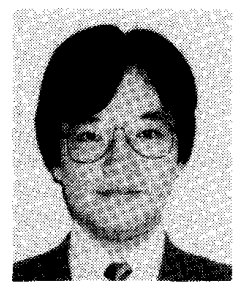

Hiroshi Mizuta (M`89) was born in Kochi, Japan, in 1961. He received the B.S. and M.S degrees in physics from Osaka University, Osaka, Japan, in 1983 and 1985 , respectively.

He joined the Central Research Laboratory, Hitachi Ltd., Tokyo, Japan, in 1985, and has been engaged in research on two-dimensional numerical simulation of heterojunction devices, and modeling of new functional devices. Since June 1989, he has been working in the field of quantum interference devices at the $\mathrm{Hi}$ tachi Cambridge Laboratory, Hitachi Europe Ltd., Cambridge, England.

Mr. Mizuta is a member of the Physical Society of Japan, the Japan Society of Applied Physics, and the Electron Devices Society of the IEEE.

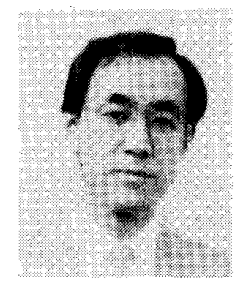

Ken Yamaguchi (M'76) was born in Tokyo, Japan, in 1947 . He received the B.Sc. and M.Sc. degrees from Yokohama National University, Yokohama, Japan, in 1970 and 1972 respectively, and the $\mathrm{Ph} . \mathrm{D}$. degree from the University of Tokyo, Tokyo, Japan, in 1980 , all in electrical engineering.

He joined the Central Research Laboratory, Hitachi Ltd., in 1972, and has been working in the field of semiconductor device design technologies. His current research interests are in device physics, device modeling, simulation technologies, and CAD systems for designing semiconductor devices and circuits.

Dr. Yamaguchi is a member of the Physical Society of Japan, the Japan Society of Applied Physics, the Institute of Electronics, Information and Communication Engineers of Japan, and the Electron Devices Society of the IEEE. 